Service social

\title{
Structures de pouvoir et services à domicile dans trois centres locaux de services communautaires.
}

Jean-Louis Gendron, Céline Archambault, Johanne Fluet et Pierre Richard

Volume 35, numéro 1-2, 1986

Recherche - Action - Évaluation

URI : https://id.erudit.org/iderudit/706302ar

DOI : https://doi.org/10.7202/706302ar

Aller au sommaire du numéro

Éditeur(s)

École de service social de l'Université Laval

ISSN

1708-1734 (numérique)

Découvrir la revue

Citer ce document

Gendron, J.-L., Archambault, C., Fluet, J. \& Richard, P. (1986). Structures de pouvoir et services à domicile dans trois centres locaux de services communautaires. Service social, 35(1-2), 214-241.

https://doi.org/10.7202/706302ar d'utilisation que vous pouvez consulter en ligne. 


\title{
COMMENTAIRES \\ ET DOCUMENTS
}

GeNDRON, Jean-Louis, professeur au Département de service social de l'Université de Sherbrooke.

ArChambault*, Céline, travailleuse sociale.

FLUET *, Johanne, travailleuse sociale.

RICHARD*, Pierre, travailleur social.

\section{Structures de pouvoir et services à domicile dans trois centres locaux de services communautaires}

\author{
Jean-Louis Gendron \\ Céline Archambault \\ Johanne Fluet \\ Pierre Richard
}

Depuis la réforme Castonguay-Nepveu, qui est à l'origine des centres locaux de services communautaires (C.L.S.C.), ces derniers ont fait l'objet de multiples études dans presque toutes les disciplines des sciences humaines ou administratives. Plusieurs d'entre elles ont été conduites au moins avec des velléités d'évaluation, sinon avec toute la stratégie de la recherche évaluative. Notre article n'échappera pas, lui non plus, à cette tendance.

Si l'un des grands objectifs des C.L.S.C., du moins à leur origine, était d'assouplir et de démocratiser les structures et la distribution des soins, notre analyse des relations de pouvoir en rapport avec les services professionnels permettra d'amorcer un bilan, de mesurer des impacts 
et, éventuellement, d'inspirer certaines corrections de parcours. Ces fonctions, qui sont essentiellement celles de la recherche évaluative, permettent de ranger notre travail dans cette catégorie, rejoignant en cela d'autres analyses qui ont contribué à l'évaluation des C.L.S.C. ${ }^{1} \mathrm{Si}$ celles-ci ont été nombreuses, il faut bien voir, cependant, que l'étude microscopique des relations de pouvoir à l'intérieur d'un C.L.S.C. paraît beaucoup moins fréquente. La science politique, en effet, se limite souvent aux grandes institutions comme les partis, les ministères ou la fonction publique, et néglige le milieu microscopique des relations de pouvoir. Certains auteurs, tels Frédéric-George Bailey (1971), Michel Crozier (1977) ou Vincent Lemieux (1978), attachent cependant une grande importance à cet aspect plus spécifique du politique.

Dans cet article, combinant une expertise en travail social et une approche théorique en science politique, nous ferons une analyse microscopique de trois programmes de services à domicile opérant dans trois C.L.S.C. différents, en tentant de faire ressortir les liens entre les structures de fonctionnement interne de ces C.L.S.C. et les services professionnels de maintien à domicile qui y sont rendus ${ }^{2}$. Notons, au départ, que les principaux bénéficiaires de ces services sont des personnes âgées.

Les C.L.S.C. étudiés ici ont été sélectionnés parce qu'ils offrent une certaine diversité quant à la taille, à leur localisation, à l'histoire de leur implantation et aux clientèles desservies. Ainsi, sans prétendre à une représentativité parfaite, notre échantillon regroupe une variété de caractéristiques intéressantes, et trois cas de C.L.S.C. assez typiques dans ce réseau d'institutions.

Géographiquement, le C.L.S.C.-X se situe dans une région excentrique où il dessert une population de 10000 habitants, dont $6 \%$ ont plus de soixante-cinq ans, et qui se répartissent dans une ville de 4500 habitants et cinq villages, le tout sur un territoire linéaire de soixante-dix kilomètres. Le C.L.S.C.-Y est situé sur un territoire qui est, en quelque sorte, la campagne immédiate d'une grande capitale régionale. Ayant son siège social dans une petite ville d'environ 1000 habitants, ce C.L.S.C. dessert aussi quelques localités plus vastes ayant des populations variant entre 1500 et 5000 habitants, de même que dix-neuf villages, pour une population totale d'environ 16000 personnes, dont plus de $20 \%$ dépasse soixante-cinq ans. Quant au C.L.S.C.-Z, il dessert un milieu essentiellement urbain et distribue des services à une population de 40000 habitants dont $12 \%$ ont plus de soixante-cinq ans ; cette population est considérée comme défavorisée aux plans économique, social et communautaire. 
Ce sont les services à domicile offerts par ces trois C.L.S.C. qui font l'objet de la présente étude. Le Ministère des affaires sociales du Québec (MAS) les a définis comme suit :

"L'ensemble des activités visant à apporter au domicile du bénéficiaire un soutien moral, matériel et médical susceptible de compenser une perte d'autonomie, de prévenir ou de pallier à une situation de crise, d'éviter ou d'abréger le séjour en institution. " ${ }^{3}$

Ce type d'aide y sera analysé à partir des pratiques quotidiennes des travailleurs sociaux et fera explicitement référence aux besoins d'entretien, d'approvisionnement et d'organisation globale de la vie quotidienne de ceux qui en bénéficient. Il s'agit donc de services concrets, quantifiables et comparables d'un C.L.S.C. à l'autre.

Cette étude est conduite sous l'angle de l'influence que peut avoir la structure organisationnelle d'un C.L.S.C. sur les pratiques professionnelles. Il s'agit donc d'une analyse politique, c'est-à-dire où la relation de pouvoir, au sein d'une organisation, constitue l'objet central de la démarche de recherche. La question qui est posée est la suivante: comment les structures de pouvoir assurant le fonctionnement interne des trois C.L.S.C. retenus influencent-elles l'organisation et la distribution des services de maintien à domicile ? Pour répondre à cette question, il faut d'abord déterminer quelle est la structure de pouvoir interne de chacun des C.L.S.C. Pour ce faire, nous empruntons à Vincent Lemieux le modèle d'analyse structurale qu'il développe dans son volume Les cheminements de l'influence ${ }^{4}$. Dans cet ouvrage, la notion de structure est très proche de celles développées par Claude Lévi-Strauss et Jean Piaget ${ }^{5}$. Ayant un caractère de totalité, une structure apparaît comme un regroupement de modèles susceptibles de se transformer en plusieurs réalisations possibles et de s'autorégler conformément à sa logique interne (Lemieux, 1979 : 117-121).

$S^{\prime}$ inspirant de la théorie des graphes orientés ${ }^{6}$, l'analyse structurale des phénomènes de pouvoir s'appuie entre autres sur les concepts de connexité et de non-connexité. Ceci fait référence à l'existence, dans un graphe donné représentant la structure d'une organisation, de chaînes ou de chemins reliant entre eux des sommets. Ces sommets représentent des acteurs qui sont, ou bien des individus, ou bien des institutions, groupes ou collectivités. Quant aux chemins, ils représentent des séquences de connexions entre les acteurs. Allant de la connexité la moins forte à la plus forte, Lemieux identifie quatre types auxquels il attribue les appellations politiques suivantes: I'anarchie, la hiérarchie, la stratarchie et la coarchie (Lemieux, 1979: 130-143). 
1. La connexité simple sera appelée anarchie. Elle est une structure de pouvoir où il n'existe pas de gouvernant, comme pourraient le représenter les graphes suivants : ${ }^{7}$

A

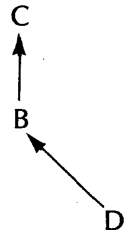

ou

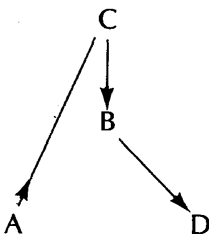

2. La connexité quasi forte sera appelée hiérarchie. C'est une structure de pouvoir faite de supérieurs et de subordonnés caractérisée par "l'existence d'un ascendant commun à deux sommets quels qu'ils soient " (Lemieux, 1979: 132). Par exemple :
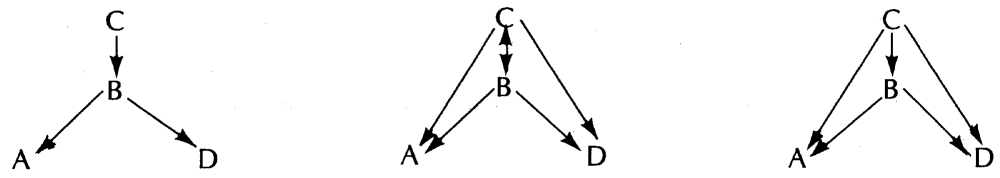

3. La connexité semi-forte sera nommée stratarchie, faisant ainsi référence à une structure de pouvoir par strates. Dans ce type de structure, "une forte proportion des participants exercent de l'influence, même si leur disposition les uns par rapport aux autres a un caractère hiérarchique" (Lemieux, 1979: 137). Visuellement, on dira qu'il y a ici existence d'au moins un chemin entre deux sommets quels qu'ils soient :
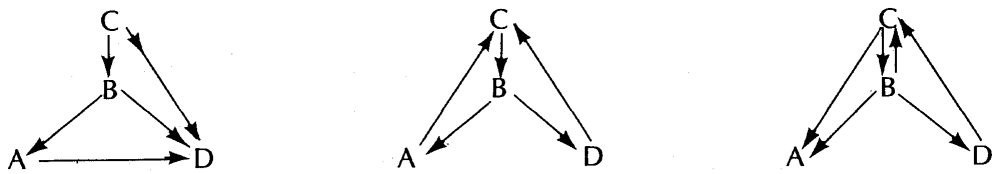

4. La connexité forte, ou coarchie, représente une structure de pouvoir égalitaire où l'influence exercée dans une direction équivaut à celle qui est appliquée dans l'autre. Il y a donc ici deux chemins reliant, en sens opposé, deux sommets quels qu'ils soient :
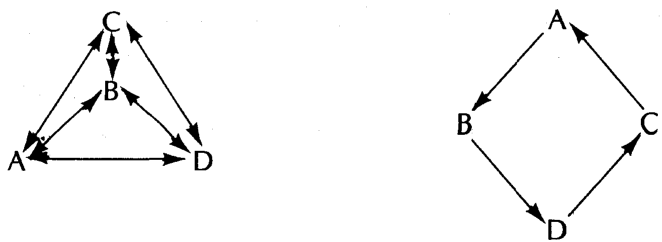


\section{TABLEAU 1}

Liste des ressources régionales en matière de services sociaux et de santé pour les trois C.L.S.C. en 1980-1981.

\begin{tabular}{|l|l|l|l|}
\hline & \multicolumn{1}{|c|}{ C.L.S.C.-X } & \multicolumn{1}{|c|}{ C.L.S.C.-Y } & C.L.S.C.-Z \\
\hline Région & rurale (périphérique) & semi-rurale (centrale) & urbaine \\
\hline Services hospitaliers & $\begin{array}{l}\text { Aucun hôpital à moins de } \\
\mathbf{4 0} \text { milles }\end{array}$ & $\begin{array}{l}\text { Aucun hôpital à moins de } \\
35 \text { milles }\end{array}$ & $\begin{array}{l}\text { Un centre hospitalier univer- } \\
\text { sitaire, trois hopitaux de soins } \\
\text { aigus et un centre hospitalier } \\
\text { de soins prolongés }\end{array}$ \\
\hline Services adaptés & - & Deux centres de jour \\
\hline $\begin{array}{l}\text { Services médicaux courants } \\
\text { autres que le C.L.S.C. }\end{array}$ & Une clinique médicale & Trois cliniques médicales & Plusieurs cliniques médicales \\
\hline $\begin{array}{l}\text { Services sociaux spécialisés } \\
\text { (C.S.S.) }\end{array}$ & $\begin{array}{l}\text { Présence d'une succursale } \\
\text { sur le territoire }\end{array}$ & $\begin{array}{l}\text { Absence d'une succursale sur } \\
\text { le territoire }\end{array}$ & $\begin{array}{l}\text { Présence d'une succursale sur } \\
\text { le territoire }\end{array}$ \\
\hline
\end{tabular}




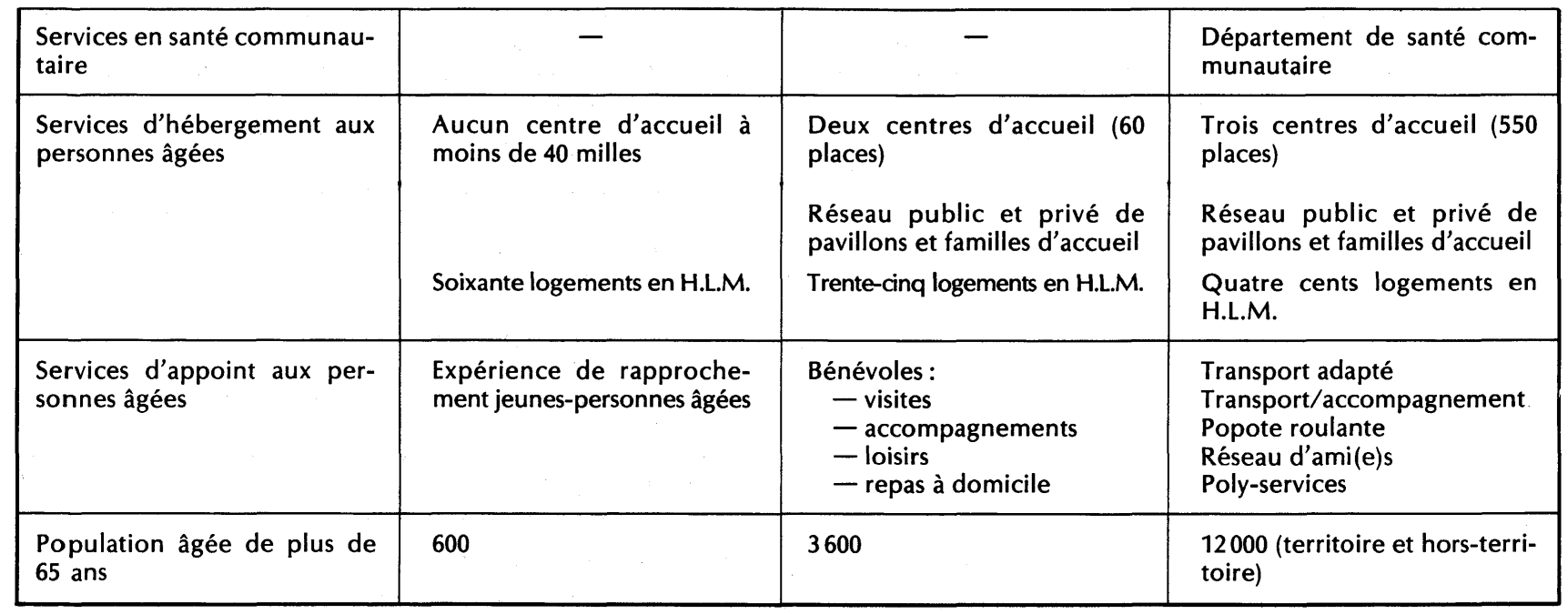


Nous utiliserons cette typologie des structures de pouvoir comme modèle théorique pour conduire l'analyse politique de nos trois C.L.S.C.

\section{Présentation des trois C.L.S.C. étudiés}

\section{Les ressources régionales}

En plus des distinctions territoriales évoquées précédemment, les trois C.L.S.C. concernés par notre étude offrent de larges différences quant à leur environnement régional, et plus particulièrement quant au réseau de ressources sociales et de santé où ils s'insèrent. Le tableau des pages 218-219 illustre clairement ces différences.

Ainsi, comme le démontre ce tableau, il existe une grande disparité de ressources en passant d'un territoire de C.L.S.C. à l'autre. La région où se situe le C.L.S.C.-Z est beaucoup plus fortement équipée que les deux autres, même si la disparité est également très grande entre le C.L.S.C.-X et le C.L.S.C.-Y.

\section{La place des programmes d'aide à domicile dans la structure des C.L.S.C.}

La recherche que nous avons faite permet de dégager globalement les structures de pouvoir qui suivent, pour chacun des CLSC ${ }^{8}$.

\section{Au C.L.S.C. $-X$}

Ce qui caractérise cet établissement, c'est son organisation par modules où des coordonnateurs appliquent les politiques émanant de la direction. Il s'agit d'une structure du pouvoir qui est, du moins officiellement, fortement hiérarchique, comme en témoigne le graphique 1.

En effet, si l'on fait exception de cet îlot coarchique que constituent les services à domicile assurés par les auxiliaires familiales et leur chef d'équipe, c'est une structure de pouvoir très fortement hiérarchique qui prévaut officiellement au C.L.S.C.-X.

\section{$A u$ C.L.S.C. $-Y$}

La principale caractéristique du mode d'organisation du C.L.S.C.-Y est que des équipes multidisciplinaires, localisées en trois points de 


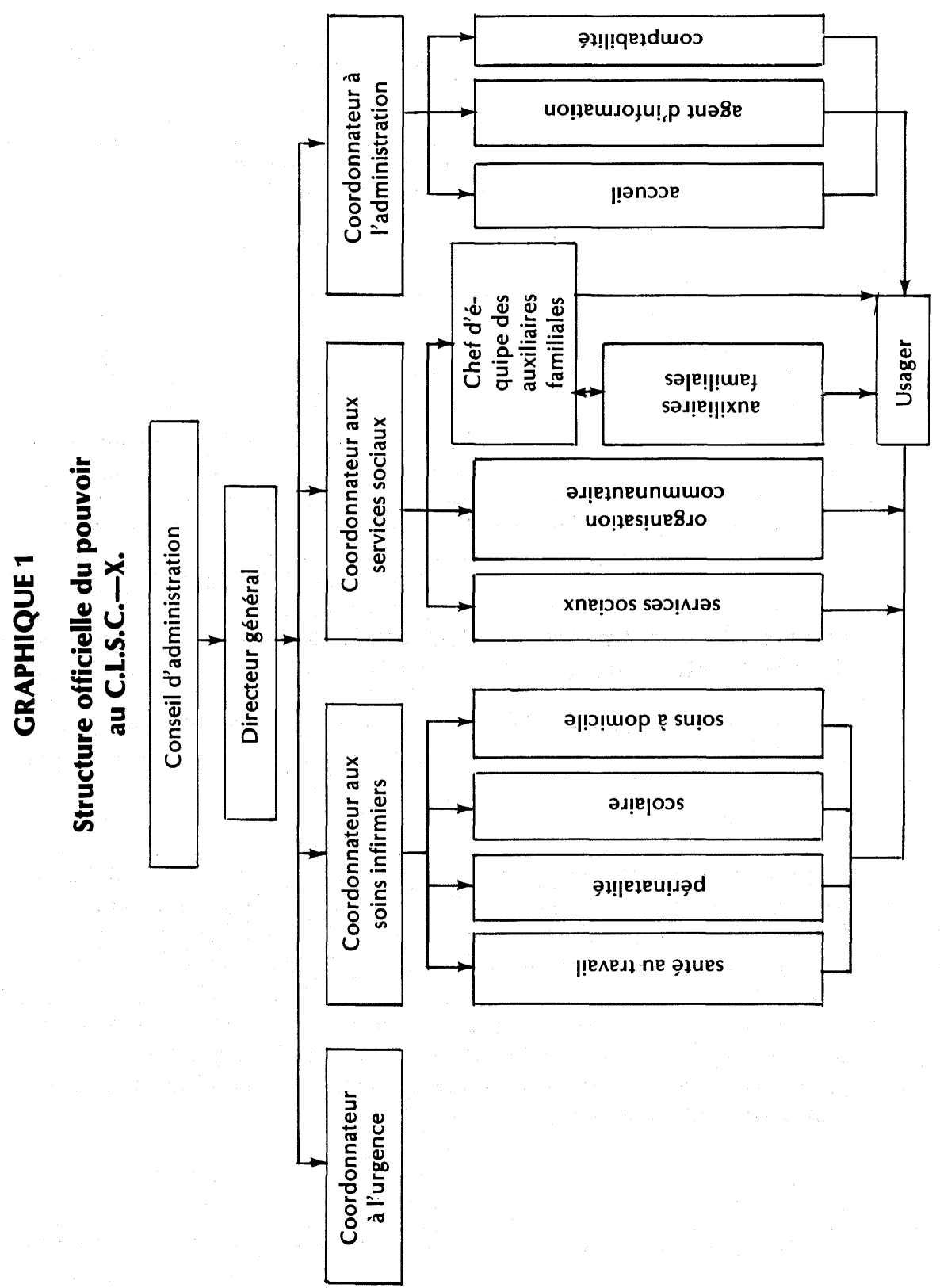




\section{GRAPHIQUE 2}

\section{Structure officielle du pouvoir au C.L.S.C. $-Y$}

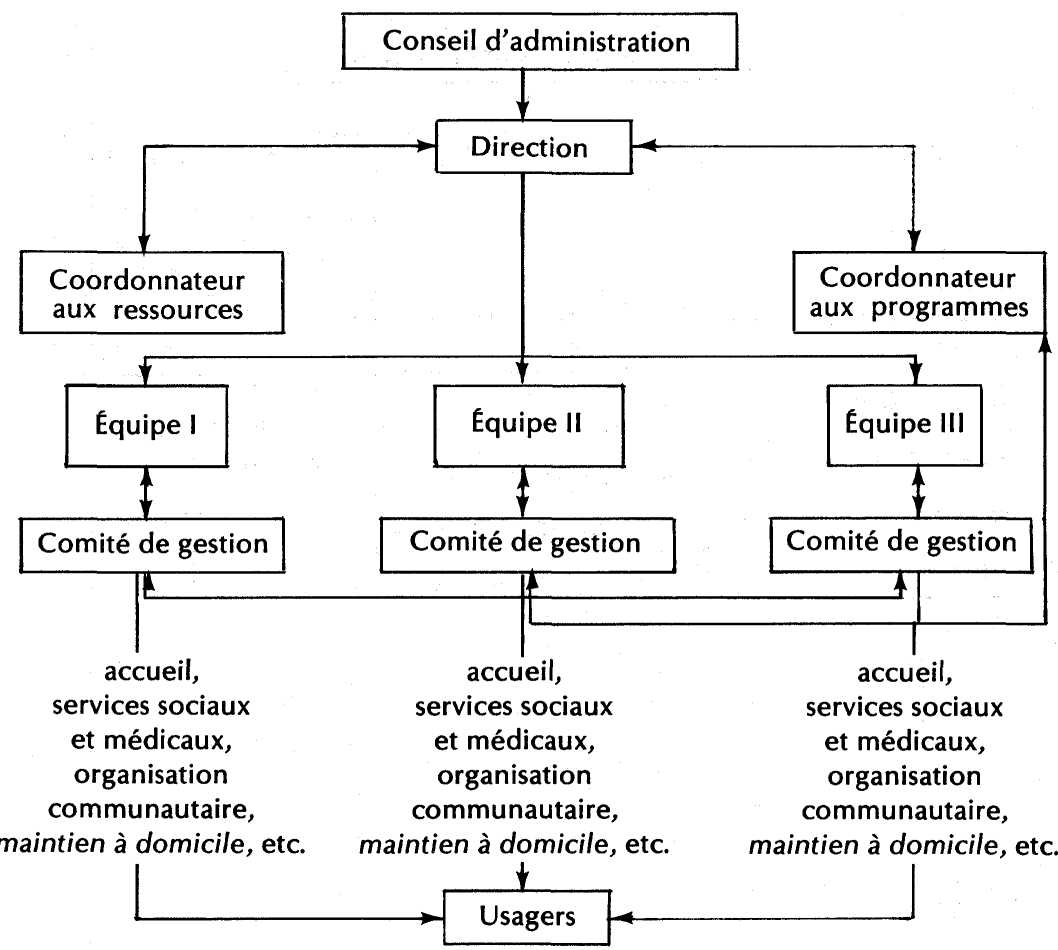

services différents, assument la dispensation de tous les services à la clientèle. Ces équipes, constituées uniquement d'intervenants, fonctionnent sans personnel-cadre et assument les tâches mentionnées au second graphique.

Nous sommes donc ici en présence d'une structure très fortement stratarchique, c'est-à-dire une organisation où une très forte proportion d'acteurs exercent de l'influence, même si leur disposition structurale les uns par rapport aux autres conserve un aspect hiérarchique. 


\section{GRAPHIQUE 3}

\section{Structure officielle du pouvoir au C.L.S.C.-Z.}

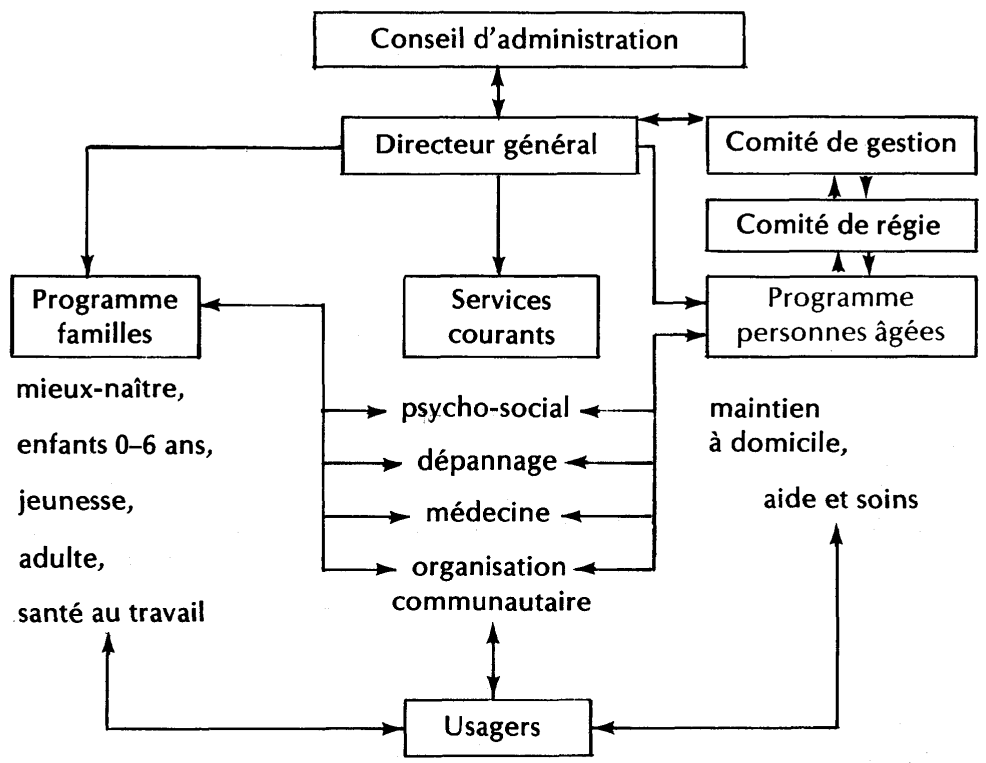

$A u$ C.L.S.C. $-Z$

Ce C.L.S.C. ${ }^{9}$ ''est toujours voulu très démocratique et proche de ses usagers. Tant par sa taille que par l'histoire de son développement, la structure officielle du pouvoir, au C.L.S.C.-Z, montre une façon différente d'organiser les services, en se basant, dans ce cas-ci, sur les types de clientèles à desservir. En examinant le graphique 3 , on constate que la direction des programmes fait le lien entre la direction générale et des services qui sont organisés tantôt sur la base de clientèles-cibles (jeunes, handicapés, etc.) et tantôt sur la base d'un service précis à offrir (maintien à domicile, dépannage, etc.). II n'est donc pas facile pour l'usager de s'y retrouver dans une telle organisation et, souvent, son seul point de repère sera l'intervenant avec lequel il entre en relation. Dans ce contexte, le maintien à domicile est un service spécifique rattaché à la population-cible des personnes âgées; comme le montre le troisième graphique. 
Tel qu'on peut le voir, la structure de pouvoir, au C.L.S.C.-Z, est beaucoup plus coarchique que celle des deux autres C.L.S.C. étudiés.

Ainsi, quelles qu'en soient les raisons, historiques ou autres, nous nous retrouvons avec trois C.L.S.C. différents, ayant chacun une structure de pouvoir qui, selon la typologie utilisée, correspond, respectivement, soit à la hiérarchie (structure autoritaire), à la stratarchie (structure stratifiée) ou à la coarchie (structure égalitaire). Mais pour répondre à notre question de recherche, existe-t-il un lien entre ces structures de fonctionnement et les services d'aide à domicile qui sont rendus dans ces C.L.S.C.?

\section{Présentation des services d'aide à domicile}

Comme nous l'avons vu, l'aide à domicile est un support moral, matériel et médical que les professionnels d'un C.L.S.C. apportent à une clientèle principalement constituée de personnes âgées, en vue tout autant d'améliorer leur qualité de vie à domicile que de repousser une entrée en centre d'accueil. Partant ici des critères d'admission, des définitions de tâche et d'un certain nombre d'autres indicateurs, nous verrons comment les trois C.L.S.C. se distinguent quant à la dispensation de cette aide à domicile.

\section{Critères d'admission, tâches professionnelles et services offerts}

Les critères d'admission aux services d'aide à domicile sont à peu près les mêmes d'un C.L.S.C. à l'autre. Ils ont trait essentiellement au degré d'autonomie, à l'isolement, à la collaboration et à l'appartenance territoriale. Tout au plus, le C.L.S.C.-Z, œuvrant en milieu urbain où les ressources sont en général davantage disponibles, a-t-il quelque peu adapté ces critères à sa réalité spécifique.

Quant aux tâches professionnelles des auxiliaires familiales qui constituent le principal personnel des services d'aide à domicile, elles se sont précisées à l'expérience et consistent maintenant en travaux ménagers légers, préparation de repas, soins d'hygiène corporelle, pratique d'exercices physiques, éducation budgétaire, ménagère ou alimentaire et support psycho-social. Ces tâches sont identiques dans les trois C.L.S.C. mais la façon de les accomplir prend une connotation préventive au C.L.S.C.-X, où l'on essaie de prévenir la détérioration des 
conditions de vie. Au C.L.S.C.-Y, et encore davantage au C.L.S.C.-Z, l'aide se limite davantage à des services minimaux et même parfois vitaux, comme la préparation de repas.

Il est difficile d'établir un lien entre ces orientations différentes et les structures de pouvoir particulières des trois C.L.S.C. Il semble bien que le ratio auxiliaires familiales/population âgée fournisse ici une meilleure explication.

\section{TABLEAU 2}

\section{Ratio auxiliaires familiales/population âgée pour les trois C.L.S.C. à l'étude.}

\begin{tabular}{|l|c|c|c|}
\hline Population & $\begin{array}{c}\text { Population } \\
\text { âgée }\end{array}$ & $\begin{array}{c}\text { Nombre } \\
\text { d'auxiliaires } \\
\text { familiales }\end{array}$ & Ratio \\
\hline C.L.S.C. & 600 & 6 & $1 / 100$ \\
C.L.S.C.-X.-Y & 3,600 & 6 & $1 / 600$ \\
C.L.S.C.-Z & 12,000 & 12 & $1 / 1000$ \\
\hline
\end{tabular}

Ce tableau montre, à l'évidence, des écarts importants entre les trois C.L.S.C. Ainsi, au C.L.S.C.-X, un usager peut recevoir les services d'une auxiliaire familiale jusqu'à dix-huit heures par semaine. Au C.L.S.C.-Y, le maximum théorique est fixé à dix heures par semaine mais, dans la pratique, on ne dépassera pas six heures. Au C.L.S.C.-Z, il est très rare que l'auxiliaire familiale se rende chez un usager pour un service de plus de trois heures par semaine et même, parfois, ce service sera de trois heures par deux semaines.

Ces ratios auxiliaires familiales/population âgée, et les disparités qui en découlent, ne peuvent prendre leur signification que s'ils sont resitués dans le contexte des ressources globales propres aux différents milieux socio-communautaires où ouvrent nos C.L.S.C.

$\mathrm{Au}$ C.L.S.C. $-X$, la première ressource est bien celle de la communauté en général qui veut que tout le monde connaisse tout le monde, si bien qu'il est fréquent de voir les gens exercer, soit une surveillance discrète sur un voisin dans le besoin, ou encore donner un coup de main en attendant un service du C.L.S.C. qui, par ailleurs, stimule et favorise cette entraide communautaire. Au C.L.S.C.-Y, des groupes de bénévoles se sont graduellement structurés, village après 
village, et ont mis sur pied des services complémentaires à ceux du C.L.S.C. Faire les courses, porter le courrier, accompagner un malade à l'hôpital sont devenus une aide précieuse, structurée et désirée par les personnes confinées à domicile et bénéficiaires, au surplus, du service d'aide à domicile. Le C.L.S.C.-Z, quant à lui, peut profiter des ressources plus abondantes d'un véritable milieu urbain. Habitations à loyer modique, service de repas à domicile ou de transport adapté, centre de services sociaux et important réseau de bénévoles complètent ses services.

Ainsi, même si la définition des services, les critères d'admission et les tâches professionnelles sont fort semblables d'un C.L.S.C. à l'autre, il est facilement compréhensible que la quantité et la qualité des services offerts varient en fonction des ressources complémentaires propres à des milieux sociaux aussi différents. Quant à la structure de pouvoir, qu'elle soit hiérarchique (C.L.S.C.-X), stratarchique (C.L.S.C.-Y) ou coarchique (C.L.S.C.-Z), son influence ne semble pas jouer aux trois niveaux étudiés ici.

\section{Perception du service d'aide à domicile par les acteurs présents dans un C.L.S.C. ${ }^{10}$}

Nous rendons compte ici de cette perception en regroupant les acteurs présents dans un C.L.S.C. selon les catégories suivantes: membres du conseil d'administration, directions générales des établissements, responsables de service, auxiliaires familiales et usagers.

\section{Les membres des conseils d'administration}

Les acteurs que nous avons interrogés dans cette catégorie sont les représentants des usagers, donc ceux qui sont les plus susceptibles de jouer un rôle ou d'avoir une opinion sur l'organisation et la distribution des services d'aide à domicile. Voici, en substance, l'opinion qu'ils ont donnée et qui vaut pour les trois C.L.S.C. :

"Au Conseil d'administration, c'est la directive du Ministère qui est importante et nous nous soucions de la faire respecter; jamais nous n'utiliserons notre pouvoir pour influencer le travail d'une auxiliaire familiale ou du responsable du service suite à des commentaires de bénéficiaires. Les besoins en aide à domicile sont manifestement grands et nous prenons bien soin que la banque d'heures autorisées soit dépensée à cette fin ; nous faisons les chiens de garde par rapport au programme des services à domicile. Nous ne croyons pas qu'il nous appartienne de nous prononcer sur les clientèles à choisir, le nombre 
d'heures à donner aux personnes dans le besoin ou même le type de service à rendre : il s'agit là du travail des responsables et des auxiliaires familiales; nous, nous surveillons l'ensemble!»

Donc, quelle que soit la structure de pouvoir dans un C.L.S.C., les représentants des usagers ne se distinguent pas des représentants des employés, du milieu ou de la direction de l'établissement. Ils forment au contraire, avec eux, une coalition qui ne se veut en position d'influencer ni la forme, ni le contenu des services d'aide à domicile. Il s'agit bien davantage d'une équipe de contrôle soucieuse de l'ensemble du C.L.S.C. et surveillant la politique du MAS quant à son application. Il y a absence totale de divergences entre les représentants des usagers et ceux de la direction ou des employés.

\section{Les directeurs généraux des établissements}

Aux questions posées, les directeurs généraux des trois C.L.S.C. ont formulé des réponses différentes.

Au C.L.S.C.-X, le directeur général se perçoit comme le gardien des politiques du MAS. En ce qui concerne les opérations concrètes du service d'aide à domicile, il s'en remet aux coordonnateurs, chefs d'équipe et auxiliaires familiales. Il établit donc une distinction entre la définition globale des objectifs et les stratégies d'intervention, montrant bien la hiérarchie qui caractérise la structure de pouvoir de son établissement.

"Par ce mode de fonctionnement, dira-t-il, nous sommes assurés du meilleur service pour la clientèle ; et les usagers peuvent influencer les services dans la mesure où ils exprimeront clairement leurs besoins pour que l'organisme puisse y répondre."

Nous avons vu, cependant, que les représentants des usagers au conseil d'administration s'en remettent eux aussi aux politiques définies par le MAS, privilégiant donc, eux aussi, une structure hiérarchique.

Au C.L.S.C. $-Y$, le directeur général perçoit l'usager comme un participant actif dans la définition et la distribution des services qu'il reçoit. De fait, le personnel de ce C.L.S.C. interprète les politiques du MAS comme des lignes directrices devant guider l'action et non comme des objectifs à atteindre. La préoccupation première du personnel est la compréhension de l'usager et l'interprétation professionnelle de ses besoins en fonction des politiques générales du MAS. II en résulte une grande souplesse de gestion laissant aux intervenants d'importantes marges de manœuvre dans l'ajustement des services aux besoins. 
Cette souplesse est encouragée par le directeur général :

"L'usager pourra influencer l'organisation et la distribution des services à la seule condition que la structure du C.L.S.C. soit suffisamment souple. II faut que la direction influence la structure de façon à ce que les usagers puissent influencer les services. "

Ce n'est donc pas par le biais de leurs représentants au conseil d'administration que les usagers sont invités à exercer leur influence, mais par le biais des professionnels avec lesquels ils sont en rapport.

Au C.L.S.C.-Z, plusieurs formes de direction se sont succédées depuis sa création en 1972. Au début, le directeur général et un comité d'usagers et d'intervenants ont conçu le service d'aide à domicile qui, par la suite, fut géré par les intervenants, ces derniers étant influencés directement et fortement par la clientèle. Les contraintes du MAS n'offraient alors qu'un encadrement normatif minimal. Au cours des dernières années, ce C.L.S.C. a vécu de nombreux soubresauts dont une mise en tutelle par le MAS. En ce qui concerne les services d'aide à domicile, il semble bien cependant que l'orientation idéologique de base ne soit plus qu'un vœu pieux, comme nous le verrons plus loin.

\section{Les responsables du service}

Dans tous les C.L.S.C., les responsables du service d'aide à domicile estiment pouvoir influencer l'organisation et la distribution des services. Il existe cependant des différences d'un C.L.S.C. à l'autre.

Au C.L.S.C. $-X$, ce responsable situe son influence exclusivement sur l'organisation et l'encadrement du travail des auxiliaires familiales. Poursuivant comme objectif d'offrir les meilleurs services possibles, il influence les auxiliaires familiales par des sessions de formation, des discussions de cas et du travail d'équipe. Cet objectif, même s'il est atteint, n'ouvre pas le C.L.S.C. à l'influence des usagers. Tout au plus les auxiliaires familiales y gagnent-elles quelque pouvoir.

Au C.L.S.C.-Y, le responsable des services à domicile se perçoit comme le gestionnaire qui planifie la distribution des ressources (heures de service disponibles) en fonction des demandes des bénéficiaires. Il estime donc pouvoir influencer le C.L.S.C. quant à la définition des besoins et à l'utilisation de ressources extérieures. D'après lui, les usagers pris un à un n'ont aucune possibilité d'influencer son service. Toutefois, les associations de bénévoles et de personnes âgées pourraient le faire, même si cela ne se produit guère.

Au C.L.S.C.-Z, c'est le responsable des services d'aide à domicile qui détermine l'attribution des services en tenant compte des 
contraintes administratives et financières du C.L.S.C. et aussi de l'achalandage des demandes en fonction de ses ressources et de celles du milieu. Il exerce donc une importante influence sur la gestion des ressources. II reconnaît pouvoir être influencé, à son tour, par les usagers, les auxiliaires familiales ou les autres intervenants, conformément aux coutumes de ce C.L.S.C., mais il ne considère pas cela comme déterminant. Ce sont les contraintes du MAS et de la structure du C.L.S.C. (pourtant coarchique) qui sont les plus fortes, selon lui.

\section{Les auxiliaires familiales}

Même si elles évaluent n'être en mesure d'influencer ni l'orientation ni les politiques de l'établissement où elles travaillent, les auxiliaires familiales des trois C.L.S.C. estiment qu'elles ont un rôle décisionnel dans la distribution immédiate des services d'aide à domicile. Elles peuvent adapter les horaires, modifier temporairement la nature d'un service, formuler des recommandations à leurs supérieurs immédiats, etc. Leur possibilité d'exercer une certaine influence à ce niveau repose sur le fait qu'elles sont très proches des bénéficiaires et capables d'interpréter correctement leurs besoins.

\section{Les usagers}

Situés à l'extrémité de la chaîne, les usagers de tous les C.L.S.C. sont convaincus de n'avoir aucun pouvoir dans l'organisation des services d'aide à domicile qu'ils reçoivent. Ils perçoivent ces organismes comme des établissements gouvernementaux complexes et loin du «monde ordinaire $"$.

Ils se disent très largement satisfaits des services qu'ils obtiennent et en accord avec les moyens utilisés pour les maintenir à domicile. Là où ils essaient de négocier des gains supérieurs, c'est avec l'auxiliaire familiale : obtenir un peu plus d'heures, un service non prévu ou « rien que pour soi». Parfois, la tentative d'influence se rendra jusqu'au responsable, sans jamais aller plus loin ni obtenir beaucoup de résultats.

De ce qui précède, il se dégage donc que les diverses catégories d'acteurs présents dans un C.L.S.C. peuvent avoir des perceptions différentes d'un service d'aide à domicile et que ces perceptions varient aussi d'un C.L.S.C. à l'autre. En effet, même si les acteurs situés aux deux extrémités de la structure de pouvoir, soit d'un côté les conseils d'administration et de l'autre les auxiliaires familiales et les usagers, ont une perception identique dans les trois C.L.S.C., les autres acteurs 
œuvrant dans la gestion des services d'aide à domicile se différencient largement en passant d'un C.L.S.C. à l'autre.

Ainsi, au C.L.S.C.-X, où une structure de pouvoir hiérarchique caractérise le fonctionnement interne de l'établissement, l'équipe des services d'aide à domicile réussit à se délimiter, à l'intérieur de cette structure, une certaine zone spécifique où elle met en place des mécanismes de collaboration à caractère fortement coarchique et où les acteurs peuvent s'influencer réciproquement. Tous ces acteurs sont cependant conscients que le pouvoir latent de la direction peut à tout moment devenir effectif pour imposer une directive ou redéfinir un objectif. Tous sont également conscients que, ni l'auxiliaire familiale ni l'usager n'ont une influence significative.

Au C.L.S.C.-Y, les normes du MAS sont connues de tous, mais ne sont pas des objectifs à atteindre. Elles sont balises et points de référence. Dès lors, le directeur général et le coordonnateur seront à coup sûr des acteurs puissants, mais leur pouvoir passera nécessairement par un comité de gestion où siègent une majorité d'employés et où se discutent les orientations de services, l'identification des clientèles cibles, les demandes de surplus budgétaires, etc. Dans ce fonctionnement stratarchique, les usagers et les auxiliaires familiales n'ont guère plus d'influence qu'au C.L.S.C.-X. Les acteurs qui profitent le plus de la stratarchie sont donc les coordonnateurs et les membres des comités de gestion.

Dans la structure théoriquement coarchique et concrètement fort complexe du C.L.S.C.-Z, c'est finalement le responsable des auxiliaires familiales qui prend toutes les décisions importantes en subissant toutefois l'influence des intervenants. Ces derniers, comme nous l'avons vu, sont des professionnels œuvrant auprès de clientèles cibles ou de services particuliers et qui interprètent les besoins des usagers au responsable de qui relève le service de maintien à domicile. Comme dans les deux autres C.L.S.C., l'influence des usagers est, à toute fin pratique, nulle, comme l'est aussi celle des auxiliaires familiales.

Il existe donc des différences reliées au mode d'organisation, au fonctionnement interne et aux rôles des acteurs qui se situent au centre de la structure de pouvoir, c'est-à-dire entre, d'une part, les conseils d'administration et, d'autre part, les usagers et les auxiliaires familiales. Mais ces différences ont-elles un rapport avec les types de structure de pouvoir qui prévalent dans chacun des C.L.S.C.?

Revenant à notre typologie des structures de pouvoir (coarchie, stratarchie, hiérarchie et anarchie), nous répondrons à cette question en examinant, pour chaque C.L.S.C., la structure de pouvoir propre de son service d'aide à domicile en resituant ce dernier à l'intérieur de 
l'ensemble de la structure de pouvoir. (Méthodologiquement, cette technique d'analyse est rendue possible grâce à un intense processus d'observation participante et à une vérification ultérieure de nos interprétations par quelques informateurs-clefs.

\section{Rôle et influence de la structure de pouvoir sur le fonctionnement des services d'aide à domicile}

Partant du fonctionnement effectif et quotidien de chaque service d'aide à domicile, nous verrons maintenant l'influence que peut avoir la structure de pouvoir du C.L.S.C. sur l'organisation et la distribution des services, répondant ainsi à notre question de recherche initiale.

\section{Le C.L.S.C. $-X$}

Comme nous l'avons vu, et si l'on s'en tient au fonctionnement quotidien, on observe à l'intérieur du service d'aide à domicile, pris séparément, une structure de pouvoir de type coarchique.

\section{GRAPHIQUE 4}

\section{Structure de pouvoir du service d'aide à domicile (pris séparément) du C.L.S.C.-X.}

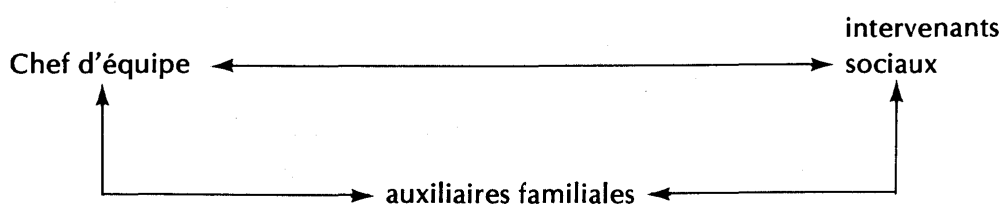

Des relations de pouvoir positif réciproques relient entre eux tous les acteurs du service d'aide à domicile, qui correspond ainsi à une structure de pouvoir coarchique. Toutefois, lorsque des relations doivent s'établir avec l'ensemble du C.L.S.C., cette structure se déplace, d'une part vers les usagers, et d'autre part vers le coordonnateur et le 
directeur général, mais sans se rendre jusqu'au conseil d'administration. Apparaît alors une structure de pouvoir de type stratarchique, différente à la fois de la structure globale du C.L.S.C. et de celle, spécifique, du service d'aide à domicile.

\section{GRAPHIQUE 5}

\section{Structure de pouvoir du service d'aide à domicile à l'intérieur du C.L.S.C.-X.}

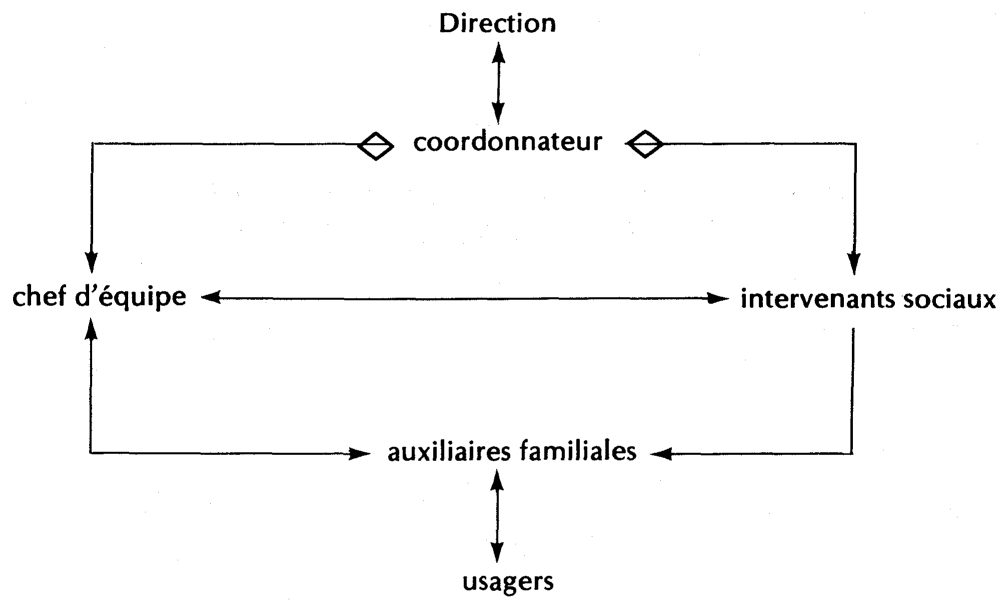

La direction intervenant toujours par le biais du coordonnateur sur les autres employés, et ces deux acteurs s'en tenant strictement aux politiques du MAS, ils forment ensemble une coalition qui les lie par une relation de pouvoir positif réciproque $(\leftrightarrow)$. Par ailleurs, leurs relations avec le chef d'équipe et le superviseur correspondent à ce que Vincent Lemieux identifie comme de la "prédominance", c'est-à-dire la possibilité pour ces acteurs, en coalition, d'exercer sur le chef d'équipe et le superviseur un pouvoir positif en même temps qu'ils sont assurés, quant à eux, de résister à l'éventuelle influence de ces derniers $(\diamond)^{11}$. Le chef d'équipe, les intervenants sociaux et, tant bien que mal, les auxiliaires familiales participent à la même coalition et, dans ces moments stratégiques, s'associent les usagers, en autant que cela leur est possible. 
Ainsi, privilégiant un fonctionnement égalitaire dans une organisation hiérarchique, le service d'aide à domicile peut stratégiquement forcer le C.L.S.C. à un certain écart par rapport à la logique de son fonctionnement global. Nous verrons plus loin que cette tendance à la coarchie sert les acteurs les moins puissants dans leurs pressions sur ceux qui détiennent plus de pouvoir.

\section{Le C.L.S.C.-Y}

Dans l'ensemble de ce C.L.S.C., la structure de pouvoir privilégiée est la stratarchie, et c'est également cette stratarchie que l'on retrouve au service de l'aide à domicile.

\section{GRAPHIQUE 6}

\section{Structure de pouvoir du service d'aide à domicile à l'intérieur du C.L.S.C.-Y.}

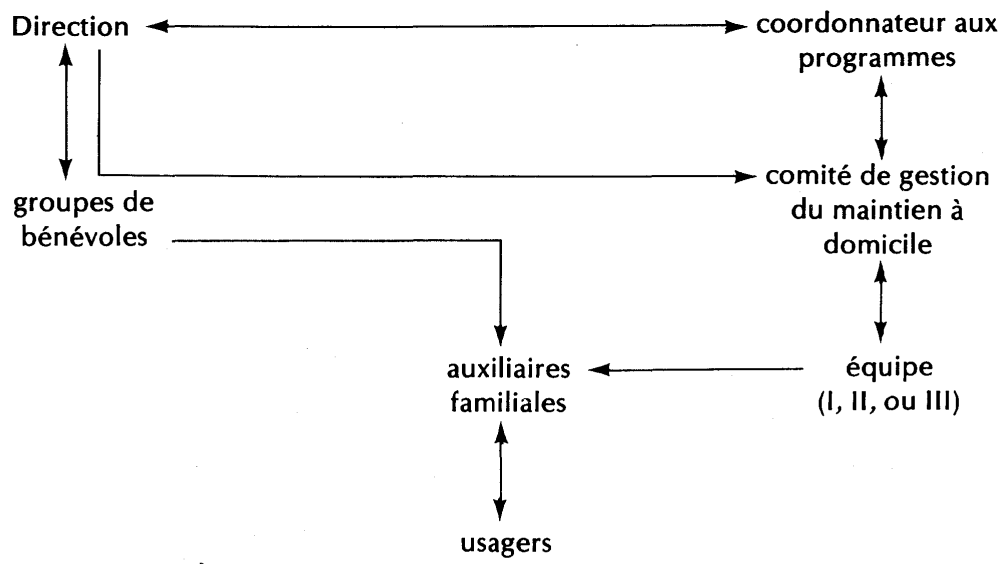

Contrairement au cas précédent, ce C.L.S.C., dans l'élaboration de ses normes, se fie moins aux directives du MAS et davantage aux services à la clientèle tels que perçus par ses agents d'intervention. Ainsi, par exemple, sur la recommandation d'un intervenant social, le conseil d'administration a déjà accepté de donner 210 heures de service 
à domicile à un usager alors que la norme du MAS n'en autorisait que 180. Le contact avec l'usager est déterminant et fait apparaître, dans la structure de pouvoir, une représentation de la population en général par des groupes de bénévoles. La stratarchie que l'on retrouve ici a une connexité plus forte qu'au C.L.S.C.-X. De fait, seuls les liens de pouvoir positif entre, d'une part, l'équipe et les auxiliaires familales et, d'autre part, entre les bénévoles et ces auxiliaires familiales, empêchent la réalisation d'une structure de pouvoir coarchique.

\section{LE C.L.S.C. $-Z$}

Ce C.L.S.C., malgré son histoire un peu tourmentée, s'est toujours réclamé d'une structure de pouvoir coarchique mais fort complexe. Dû probablement à cette complexité, et si l'on veut prendre en compte tous les acteurs qui participent au processus décisionnel en ce qui touche le service d'aide à domicile, on constate que les usagers et les auxiliaires familiales sont en pratique incapables d'exercer une influence réelle sur la structure de pouvoir de leur service; ceci est dû à la résistance du responsable de l'aide à domicile, et ce, malgré l'appui réciproque des autres professionnels.

En effet, cette structure de pouvoir de l'équipe de l'aide à domicile prend la configuration suivante :

\section{GRAPHIQUE 7}

\section{Structure de pouvoir du service d'aide à domicile à l'intérieur du C.L.S.C.-Z.}

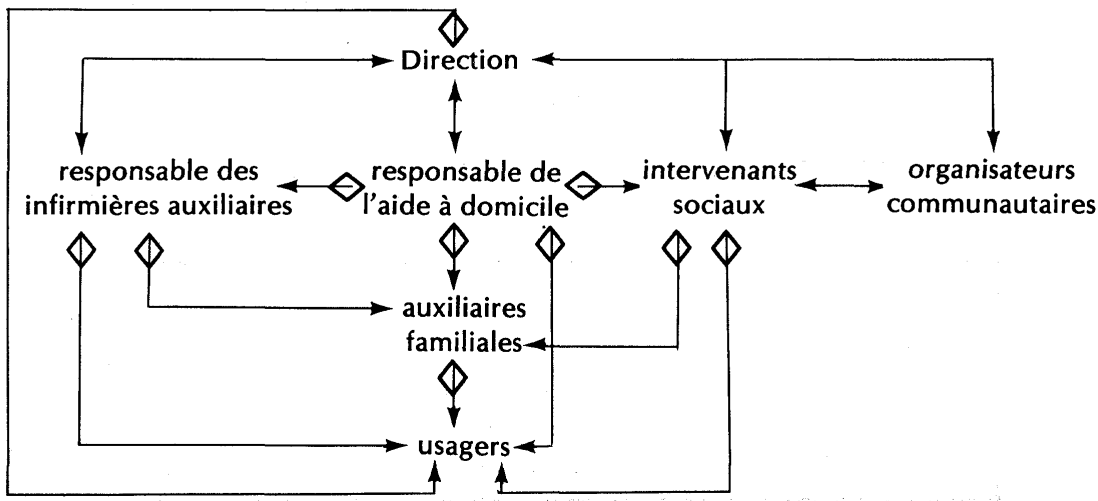


La direction du C.L.S.C., en plus d'exercer sa prédominance sur les usagers, forme trois coalitions distinctes, l'une avec le responsable des infirmières auxiliaires, l'autre avec le responsable de l'aide à domicile et la troisième avec les intervenants sociaux et les organisateurs communautaires. Ces trois coalitions exercent chacune cette prédominance sur les auxiliaires familiales et les usagers. Nous nous retrouvons donc avec une structure de pouvoir hiérarchique prenant la configuration suivante, si nous la simplifions :

\section{GRAPHIQUE 8}

\section{Configuration simplifiée du pouvoir au service d’aide à domicile du C.L.S.C. $-Z$.}

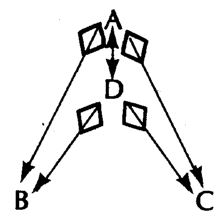

$$
\begin{aligned}
& A=\text { direction générale } \\
& D=\text { responsable de l'aide à domicile } \\
& B=\text { auxiliaires familiales } \\
& C=\text { usagers }
\end{aligned}
$$

Paradoxalement, dans ce C.L.S.C. qui se veut coarchique pour l'ensemble de sa structure, le service d'aide à domicile fonctionne avec une structure de pouvoir fortement hiérarchique.

Si l'on résume donc, dans un tableau, les structures de pouvoir observées dans l'ensemble de chacun des C.L.S.C. et dans leurs services d'aide à domicile respectifs, on en arrive au résultat qui suit.

\begin{tabular}{|c|c|c|}
\hline C.L.S.C. & $\begin{array}{l}\text { Structure } \\
\text { d'ensemble }\end{array}$ & $\begin{array}{l}\text { Structure de l'aide } \\
\text { à domicile }\end{array}$ \\
\hline $\begin{array}{l}X \\
Y \\
Z\end{array}$ & $\begin{array}{l}\text { hiérarchique } \\
\text { stratarchique } \\
\text { coarchique }\end{array}$ & $\begin{array}{l}\text { coarchique } \\
\text { stratarchique } \\
\text { hiérarchique }\end{array}$ \\
\hline
\end{tabular}

\section{TABLEAU 3}

Structures de pouvoir comparées entre les trois C.L.S.C. et leurs services d'aide à domicile. 
Rappelons que la hiérarchie réfère à une structure de pouvoir linéaire faite de supérieurs et de subordonnés; que la stratarchie se retrouve lorsqu'une forte proportion de participants exercent de l'influence, même si la structure de pouvoir conserve en partie sa configuration hiérarchique ; et que la coarchie représente l'égalitarisme des relations.

Dans le cas des C.L.S.C. à l'étude, on constate qu'une structure globalement hiérarchique a permis à la coarchie de se développer en son sein alors, qu'à l'inverse, une structure hiérarchique a pris racine dans un C.L.S.C. coarchique. Seul le C.L.S.C.-Y demeure stratarchique à tous les niveaux.

Ces différentes configurations du pouvoir ne sont pas sans nous rappeler la loi du jeu politique énoncée par Vincent Lemieux dans Les cheminements de l'influence. En effet, tel que le veut cette loi du politique, dans les jeux d'influence auxquels s'adonnent les acteurs présents dans une organisation, "les acteurs les plus puissants tendent à la hiérarchie, tout en préférant la stratarchie à la coarchie ; les acteurs dont la puissance est moyenne tendent à la stratarchie, tout en préférant l'anarchie à la hiérarchie, et les acteurs les moins puissants tendent à la coarchie, tout en préférant la hiérarchie ou même l'anarchie à la stratarchie $» .12$

Au C.L.S.C.-X, la structuration officielle de pouvoir est hiérarchique. Les acteurs rattachés au service d'aide à domicile sont les moins puissants et, comme nous l'avons vu, ils tendent très nettement à privilégier la coarchie, cette structure de pouvoir les assurant d'exercer une pression plus forte sur leur coordonnateur et leur directeur général. L'influence de la structure de pouvoir sur la distribution des services se limite cependant à cette dynamique interne. Tel que nous l'avons vu, le conseil d'administration ne s'implique pas à ce niveau, et les auxiliaires familiales de même que les usagers ont une implication plus stratégique que volontaire.

Au C.L.S.C. $-Y$, les structures officielles de pouvoir sont stratarchiques et les intervenants du service d'aide à domicile recherchent le maintien de cette structure dans le processus de distribution des services. Encore ici, cependant, malgré une intégration du directeur général à cette stratarchie, le conseil d'administration, lui, y demeure absent, alors que les usagers et les auxiliaires familiales ne s'imbriquent que difficilement dans cette structure. Comme dans le cas précédent on peut donc constater que la structure de pouvoir influence la distribution des services chez les acteurs qui se situent au centre du système (intervenant et directeur général), mais laissent de côté les acteurs qui sont à la périphérie (auxiliaires familiales, usagers et conseil d'administration). 
Au C.L.S.C.-Z, la recherche globale de coarchie existe toujours malgré la tutelle qui pèse sur lui au moment de l'étude. Indépendamment de cette tutelle, et devant la complexité de la structure, nous avons vu le caractère hiérarchique du service d'aide à domicile. Apparaissant comme un acteur très puissant dans l'ensemble de cette organisation, le service d'aide à domicile privilégie la hiérarchie qui lui permet une argumentation plus forte dans l'ensemble du C.L.S.C. Encore ici, cependant, les usagers, les auxiliaires familiales et le conseil d'administration ne perçoivent guère la différence entre une structure hiérarchique ou coarchique, au service d'aide à domicile.

\section{Conclusion}

À quelles conclusions faut-il donc en venir, si l'on veut répondre à notre interrogation de départ, à savoir l'influence des structures de fonctionnement interne des C.L.S.C. sur les services professionnels de maintien à domicile qui y sont rendus?

La première conclusion qu'il faut tirer de cette étude est que les normes et directives émises par le MAS sont d'une telle étanchéité que les structures de pouvoir officielles des C.L.S.C. ne peuvent guère varier, si l'on y inclut les conseils d'administration et les usagers. Ces deux derniers acteurs ont une place identique dans la structure de pouvoir des trois C.L.S.C.: les conseils d'administration se présentent uniformément comme responsables de l'application des directives du MAS et les usagers comme bénéficiaires de ces directives.

La deuxième conclusion, corollaire de la précédente, est à l'effet que seuls les agents de gestion internes peuvent profiter d'une certaine marge de manœuure dans l'application des normes du MAS. Et c'est seulement ici que l'on peut observer un lien précis entre les structures de fonctionnement interne et les services professionnels. Le fonctionnement coarchique du service d'aide à domicile du C.L.S.C. $X$ ne change pas grand-chose aux services offerts à l'usager, mais donne plus de légitimité au chef d'équipe; au C.L.S.C.-Y, les usagers et les auxiliaires familiales sont isolés de la prise de décision, et la structure stratarchique leur permet tout au plus de faire pression pour l'obtention ou la modification d'un service; au C.L.S.C.-Z, le gestionnaire du service accepte les demandes, évalue les besoins et détermine les services, alors que les usagers et les intervenants se limitent à présenter leurs besoins. Donc, si les choses varient d'un C.L.S.C. à l'autre, c'est strictement dans cette zone institutionnelle 
intermédiaire où se situe le personnel de gestion du service; ni les usagers, ni les conseils d'administration ne sont touchés.

Dans les faits, bien plus que la structure de pouvoir c'est la qualité du fonctionnement quotidien d'un service qui influence sa distribution : tout repose sur la compétence, la disponibilité et la bonne volonté de son responsable, ces valeurs n'ayant rien à voir avec la hiérarchie, la stratarchie ou la coarchie des structures. C'est ce qui permet d'expliquer que l'usager passe davantage par les relations personnelles que par la structure pour formuler ses demandes.

Mais cet usager, qui est la raison d'être de ces services, qu'en restet-il dans ces structures? Il faut noter très clairement que l'usager des services à domicile, quoi qu'en aient déjà dit les discours officiels, ne fait pas partie de la structure organisationnelle d'un C.L.S.C. : il reçoit des services et les consomme. Rares sont les personnes confinées à domicile et qui peuvent encore siéger sur des comités ou à un conseil d'administration de C.L.S.C. C'est seulement lors de la réception d'un service qu'il peut tenter d'exercer son influence. Pour lui, les C.L.S.C. sont des appareils d'État où les groupes populaires qui les ont promus n'ont plus grand-chose à dire. Comme nous l'avons vu, il se rabat donc sur la seule possibilité qui lui reste: influencer personnellement l'auxiliaire familiale qui lui apporte un service, tenter d'allonger son temps de travail auprès de lui, changer son horaire, etc. Et, ici encore, l'influence effective de l'usager se mesure bien davantage par l'ouverture d'esprit du personnel de C.L.S.C. que par la structure de pouvoir interne.

Or, les deux C.L.S.C. de milieux ruraux ( $\mathrm{X}$ et $\mathrm{Y}$ ) œuvrent dans des contextes sociaux où les réseaux traditionnels de voisinage, de parenté ou d'amitié sont beaucoup plus importants qu'en milieu urbain (C.L.S.C.-Z). Dès lors, la connaissance quasi personnelle de tout un chacun dans ces réseaux influence l'auxiliaire familiale et même ses chefs de service de telle façon que la pression exercée par un usager a souvent plus d'influence que dans l'anonymat d'un milieu urbain. Cet environnement social, différent pour chaque C.L.S.C., n'est certainement pas indifférent au fait que les usagers aient affirmé, en entrevue, avoir plus de chances d'exercer leur influence au C.L.S.C.-X (stratarchique) et au C.L.S.C.-Y (hiérarchique) qu'au C.L.S.C.-Z, qui, lui seul, se veut coarchique.

Dans trois C.L.S.C., éloignés géographiquement et socialement l'un de l'autre mais soumis aux mêmes normes gouvernementales, nous avons étudié trois services d'aide à domicile; ceux-ci appliquent les mêmes directives émanant de ces normes et se dotent de structures de pouvoir différentes quant au fonctionnement interne des acteurs 
chargés d'appliquer ces directives; les clientèles des C.L.S.C. ne voient pas de différence entre les services rendus, que la structure du pouvoir soit coarchique, stratarchique ou hiérarchique.

\section{Notes et références}

* Céline Archambault, Johanne Fluet et Pierre Richard étaient tous trois étudiants au Département de service social de l'Université de Sherbrooke au moment de la production de l'essai de maîtrise d'où est tiré le présent article.

1 Pour n'en mentionner que quelques-uns, rappelons les travaux de : Marc Renaud (1976), Jacques Alary et Frédéric Lesemann (1975), Vincent Lemieux $(1975,1977,1979)$ et Jean-Louis GeNDRON (1979).

2 La cueillette des données a été effectuée en 1980-1981. Le rapport de recherche, présenté en août 1985, constituait le mémoire de maîtrise de trois étudiant(e)s du Département de service social de l'Université de Sherbrooke. Une vérification sommaire dans les trois C.L.S.C. au moment de la préparation de cet article permet de croire que la situation est actuellement assez semblable à celle qui prévalait au moment de la cueillette des données, du moins dans deux cas sur trois. Quant au troisième cas, il est vraisemblable que sa structure de pouvoir se soit quelque peu hiérarchisée. La problématique des relations de pouvoir, en rapport avec la distribution des services, reste cependant largement semblable.

3 Ministère des affaires sociales, Les services à domicile : politique du Ministère des affaires sociales, Québec, 1979, p. 7.

4 V. LemieuX, Les cheminements de l'influence, Québec, PUL, 1979, pp. 116-143.

5 C. LÉvi-Strauss, Anthropologie structurale, Paris, Plon, 1958, p. 306. J. Piaget, Le structuralisme, Paris, PUF, 1969, pp. 6-7.

6 F. HaRARY et al., Introduction à la théorie des graphes orientés, Paris, Dunod, 1968.

7 Dans tous les graphes qui suivent, les lettres représentent des acteurs présents dans une organisation et les flèches représentent des relations de pouvoir. La capacité d'un acteur à en faire agir un autre (pouvoir positif) est représentée par le symbole suivant: $\rightarrow$; la possibilité pour un acteur de résister à l'influence d'un autre (pouvoir négatif) est représentée par le symbole suivant $: \multimap$.

8 La cueillette des données nécessaires à cette recherche a été effectuée entre novembre 1981 et septembre 1982 par trois travailleurs sociaux œuvrant dans chacun des C.L.S.C. Leur statut d'observateur-participant représentait un avantage évident, mais aussi un risque certain d'y introduire certains biais. Pour limiter ces biais, un total de trente-huit entrevues semi-dirigées ont été conduites auprès des conseils d'administration, directeurs généraux, coordonnateurs de programmes, responsables de services, auxiliaires familiales et usagers des trois C.L.S.C. Ce nombre de trente-huit a été fixé conformément à la technique de "saturation des catégories" (GLASER et Strauss, 1967). De plus, un premier rapport de recherche a été largement diffusé auprès de ces différentes catégories d'acteurs de façon à ce que leurs 
réactions puissent être enregistrées et utilisées pour rectifier certains faits qui auraient pu avoir été mal compris ou mal interprétés par les chercheurs.

9 Rappelons que la situation décrite ici correspond aux réalités de 1980, immédiatement avant sa mise en tutelle. La situation actuelle pourrait s'avérer un peu différente et tendre vers la stratarchie.

10 Dans la section précédente, portant sur les critères d'admission, les tâches et les services, la cueillette des données a été effectuée à l'aide, principalement, de l'analyse documentaire et de l'observation participante. Pour la présente section, portant sur les perceptions des acteurs, nous avons eu recours à l'entrevue centrée auprès de nos trente-huit acteurs (clients, auxiliaires familiales, travailleurs sociaux, cadres, etc.) qui ont eu à répondre aux deux questions suivantes:

1) Croyez-vous que vous avez un rôle à jouer dans l'organisation et la distribution des services d'aide à domicile?

2) Croyez-vous que les usagers ont un rôle à jouer dans l'organisation et la distribution des services d'aide à domicile?

11 Vincent LEMIEUX, op. cit., pp.51-52. Il s'agit de la combinaison de deux relations de pouvoir positif $\leftrightarrows$ et d'une relation de pouvoir négatif ce qui donne $\leftrightarrows$ ou $\diamond$.

12 Vincent Lemieux, op. cit., pp. 161-162.

\section{Bibliographie}

Alarr, J. et F. LESEMANN, Étude des dimensions sociales et communautaires, Opération Bilan, Montréal, (miméo), 1975.

Archambault, C., J. Fluet et P. Richard, Étude comparée de l'exercice du pouvoir dans trois C.L.S.C. québécois, essai de maîtrise, Département de service social, Université de Sherbrooke, 1985.

Balley, F.-G., Les règles du jeu politique, Paris, PUF, 1971.

Crozier, M. et E. FriedberG, L'acteur et le système, Paris, Seuil, 1977.

Gendron, J.-L., La structuration du pouvoir dans l'implantation des C.L.S.C. en Estrie, thèse de doctorat, Université Laval, 1979.

Glaser, B. et A. Strauss, The Discovery of Grounded Theory: Strategies for Qualitative Research, Chicago, Aldine, 1967.

Harary, F. et al., Introduction à la théorie des graphes orientés, Paris, Dunod, 1968.

Lemieux, V., Les conseils régionaux de la santé et des services sociaux: une analyse politique, (miméo). Département des sciences politiques, Université Laval, Québec, 1975.

Lemieux, V., Les cheminements de l'influence, Québec, PUL, 1978.

Lemieux, V. et R. Fortin, "Réseaux et appareils dans l'implantation de deux C.L.S.C. ", Recherches sociographiques, vol. 28, n² 2, 1977 : 177-190.

LEMIEUX, V. et P. LABRIE, "Le système gouvernétique des CLSC", Recherches sociographiques, vol. $20, \mathrm{n}^{\circ} 2,1979: 149-171$. 
LÉvi-Strauss, C., Anthropologie structurale, Paris, Plon, 1958.

Piaget, J., Le structuralisme, Paris, PUF, 1969.

Renaud, M., The Political Economy of the Quebec State Interventions in Health: Reform or Revolution?, thèse de doctorat, Université du Wisconsin, 1976. 